\title{
Phase II, open-label, multicenter trial of crizotinib in Japanese patients with advanced non-small cell lung cancer harboring a MET gene alteration: Co-MET study
}

Mototsugu Shimokawa ${ }^{1,2^{*}}$, Kaname Nosaki ${ }^{3,4}$, Takashi Seto $^{3}$, Kadoaki Ohashi $^{5}$, Masahiro Morise ${ }^{6}$, Hidehito Horinouchi ${ }^{7}$, Jun Sakakibara ${ }^{8}$, Haruyasu Murakami ${ }^{9}$, Seiji Yano ${ }^{10}$, Miyako Satouchi ${ }^{11}$, Shingo Matsumoto ${ }^{4}$, Koichi Goto ${ }^{4}$ and Kiyotaka Yoh ${ }^{4}$

\begin{abstract}
Background: MET-deregulated non-small cell lung cancer represents an urgent clinical need because of the lack of specific therapies. Although recent studies have suggested a potential role for crizotinib in patients harboring MET gene alterations, no conclusive data are currently available. Therefore, we designed the Co-MET study, a single-arm phase II study to assess the efficacy and safety of crizotinib in patients with advanced non-small cell lung cancers harboring MET gene alterations.

Methods: Co-MET is an open-label, multi-center, single-arm, phase II trial to assess the safety and efficacy of oral crizotinib in patients with advanced non-small cell lung cancer harboring MET exon 14 skipping mutation (cohort 1) or a high MET gene copy number of $\geq 7$ (cohort 2). We will identify MET gene alterations using RT-PCR and/or nextgeneration sequencing. Oral crizotinib $250 \mathrm{mg}$ BID will be administered until disease progression or unacceptable toxicity. A radiology committee will review tumor scans according to the RECIST criteria. The primary endpoint is the objective response rate. Assuming a null hypothesis of $20 \%$ objective response rate and an alternative hypothesis of $50 \%$ objective response rate for cohort 1, and a one-sided alpha error of 0.05 and $80 \%$ power based on the exact binomial distribution, the required number of evaluable patients is 19 . We set the exploratory sample size for cohort 2 at 10 patients.
\end{abstract}

Discussion: The results of this study are expected to provide evidence regarding the usefulness of oral crizotinib for advanced MET exon 14 skipping mutation-positive or MET high gene copy number-positive non-small cell lung cancer.

Trial registration: This study was registered with the University Hospital Medical Information Network Clinical Trials Registry as UMIN000031623 on 3 March 2018.

Keywords: Non-small cell lung cancer, Crizotinib, MET gene alteration, RT-PCR assay, Next-generation sequencing

\footnotetext{
* Correspondence: moto@yamaguchi-u.ac.jp

${ }^{1}$ Cancer Biostatistics Laboratory, Clinical Research Institute, National Hospital Organization Kyushu Cancer Center, Fukuoka, Japan

2Department of Biostatistics, Yamaguchi University Graduate School of

Medicine, 1-1-1 Minamikogushi, Ube, Yamaguchi 755-8505, Japan

Full list of author information is available at the end of the article
}

(c) The Author(s). 2020 Open Access This article is licensed under a Creative Commons Attribution 4.0 International License, which permits use, sharing, adaptation, distribution and reproduction in any medium or format, as long as you give appropriate credit to the original author(s) and the source, provide a link to the Creative Commons licence, and indicate if changes were made. The images or other third party material in this article are included in the article's Creative Commons licence, unless indicated otherwise in a credit line to the material. If material is not included in the article's Creative Commons licence and your intended use is not permitted by statutory regulation or exceeds the permitted use, you will need to obtain permission directly from the copyright holder. To view a copy of this licence, visit http://creativecommons.org/licenses/by/4.0/ The Creative Commons Public Domain Dedication waiver (http://creativecommons.org/publicdomain/zero/1.0/) applies to the data made available in this article, unless otherwise stated in a credit line to the data. 


\section{Background}

Non-small cell lung cancer (NSCLC) is a common cause of cancer mortality worldwide. The histological diagnoses include $\sim 85 \%$ of non-small cell and $\sim 15 \%$ of cell lung cancers. The majority of patients with NSCLC have a metastatic disease at diagnosis, for which no curative treatment exists. Platinum-based chemotherapies were standard for patients with NSCLC and good performance status. Phase III randomized trials of tyrosine kinase inhibitor (TKI) therapy for EGFR-mutant and anaplastic lymphoma receptor tyrosine kinase (ALK)-rearranged lung cancers have shown documented improvements in response and progression-free survival (PFS) [1-3], and TKIs are approved for patients with oncogene-driver mutations. NSCLC represents a paradigm for the development of targeted cancer therapy. Advancements in nextgeneration sequencing (NGS) technology have greatly assisted the discovery of rare driver mutations that may serve as potential therapeutic targets in lung cancer $[4,5]$.

c-Met is the tyrosine kinase receptor for hepatocyte growth factor (HGF). Binding of HGF to MET stimulates downstream signal pathways, such as the RAS/ERK/ MAPK, PI3K/AKT, Wnt//-catenin, and STAT signaling pathways. These pathways are known to involve cell growth, migration, angiogenesis, and survival. MET gene alterations, including MET exon 14 skipping mutationpositive or MET high gene copy number, generate oncogenes via activation of c-MET signaling pathway $[5,6]$.

Crizotinib is a selective ATP-competitive small-molecule inhibitor of c-Met, ALK, and ROS1 (c-ros) tyrosine kinases. Dramatic and durable responses to crizotinib were first reported in mid-2015 in patients with advanced NSCLC harboring MET exon 14 skipping mutation [7-9]. Crizotinib also demonstrated efficacy in NSCLC with high MET gene copy number $[10,11]$.

Since MET-deregulated NSCLC represents an urgent clinical need because of a lack of approved specific therapies, we designed a trial to assess the efficacy and safety of crizotinib in patients with advanced NSCLCs harboring MET gene alterations. The planned protocol will include 29 response-evaluable patients with advanced NSCLC whose tumors contain MET exon 14 skipping mutations or high MET gene copy number. We will use a validated reverse transcription polymerase chain reaction (RTPCR) and/or NGS assay (multi-screening using oncomine comprehensive assay (OCA) panel) to identify MET gene alterations.

\section{Methods/Design \\ Study design and objective}

This is an open-label, multi-center, two cohort, singlearm, phase 2 trial of oral crizotinib in patients with advanced NSCLC harboring MET exon 14 skipping mutation (cohort 1) or high MET gene copy numbers of seven or more (cohort 2) (Fig. 1). The population in cohort 1 includes patients with advanced NSCLC harboring MET exon 14 skipping mutation who have not received prior MET inhibitor treatments. We will confirm MET gene alterations using a validated RT-PCR and/or NGS assay. Our primary objective is to assess the antitumor activity of oral single-agent crizotinib for advanced MET exon 14 skipping mutation-positive or MET high gene copy number-positive NSCLCs as measured by the objective response rate (ORR). Our secondary objectives included assessing secondary measures of antitumor activity by duration of response (DR); disease control rate at 8,16 , and 24 weeks; and PFS and overall survival and the safety and tolerability of oral crizotinib. This study will be conducted according to the standards of Good Clinical Practice and in compliance with the principles of the Declaration of Helsinki, and our Institutional Review Board has approved the protocol registered in the University Hospital Medical Information Network Clinical Trials Registry as UMIN000031623 (https://upload.umin.ac.jp/

\section{Co-MET study schema (Phase 2)}

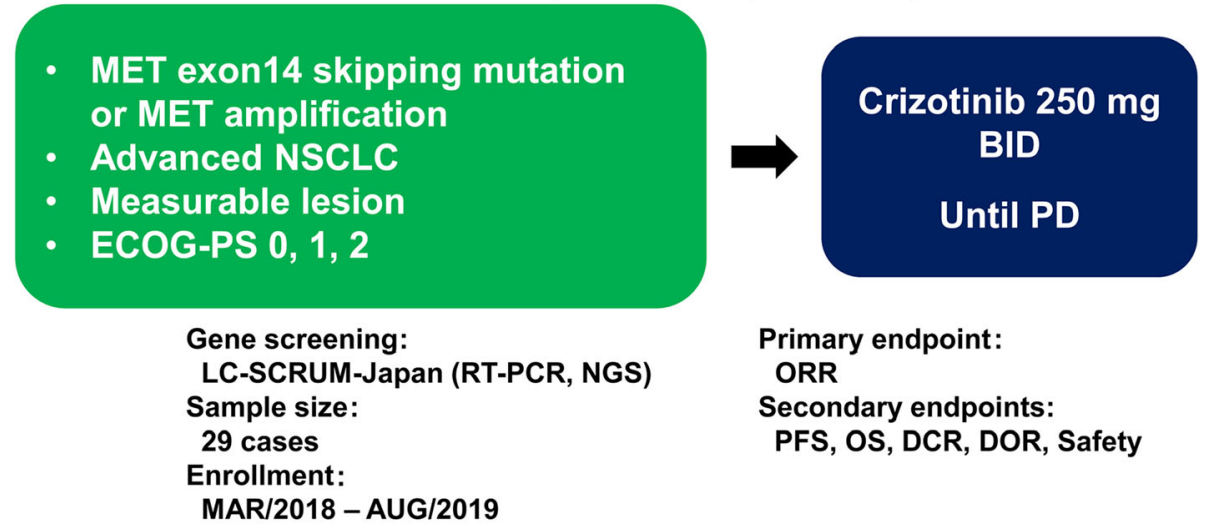

Fig. 1 Co-MET study schema (phase 2) 
Table 1 Inclusion and exclusion criteria

Inclusion criteria
1. Histologically or cytologically confirmed NSCLC that is locally
advanced or metastatic
2. Positivity for MET exon 14 skipping mutation or high MET copy
number (seven or more) as determined by a validated RT-PCR
and/or NGS assay by a designated central testing laboratory
(LC-SCRUM)
*Patients positive for MET exon 14 skipping mutation and high
MET copy number of seven or more will be enrolled in cohort 1.

3. At least one measurable tumor lesion as per RECIST (version 1.1) that has not been irradiated

4. Women or men, 20 years of age or older

5. ECOG performance status between 0 and 2

6. Adequate organ function as defined by the following criteria:

Serum aspartate transaminase (AST) and serum alanine transaminase (ALT) $<2.5 \times$ upper limit of normal (ULN), or AST and ALT $<5 \times$ ULN if liver function abnormalities are due to underlying malignancy

Total serum bilirubin $<1.5 \times$ ULN

Absolute neutrophil count (ANC) $>1500 / \mu \mathrm{L}$

Platelets $>50,000 / \mu \mathrm{L}$

Hemoglobin $>8.0 \mathrm{~g} / \mathrm{dL}$

Serum creatinine $<2 \times$ ULN

7. Signed and dated informed consent document indicating that the patient has been informed of all the pertinent aspects of the trial prior to enrollment

8. Willingness and ability to comply with scheduled visits, treatment plans, laboratory tests, and other study procedures

9. Agreement to use effective contraception during the study period and for at least 90 days after the last dose of crizotinib

Exclusion criteria

1. Current treatment on another therapeutic clinical trial

2. Characterized ALK or ROS1-positive rearrangement

3. Prior therapy specifically directed against MET

4. Any treatment (chemotherapy, radiation, or surgery) within 2 weeks prior to study entry, except for patients who completed palliative radiation $48 \mathrm{~h}$ prior to study entry

5. Any acute toxicity $>$ Grade 1

6. Symptomatic brain metastases. Eligible if asymptomatic, or if treated (must be neurologically stable for at least 2 weeks and are not taking unstable or increasing doses of corticosteroids)

7. Spinal cord compression unless treated with the patient attaining good pain control and stable or recovered neurologic function, carcinomatous meningitis, or leptomeningeal disease

8. Known interstitial fibrosis or interstitial lung disease

9. Any of the following within the 3 months prior to starting study treatment: myocardial infarction, severe/unstable angina, coronary/peripheral artery bypass graft, congestive heart failure, or cerebrovascular accident including transient ischemic attack

10. Ongoing cardiac dysrhythmias of NCI CTCAE v4.03 Grade > 2, uncontrolled atrial fibrillation of any grade, or QTC $>470 \mathrm{msec}$

11. Pregnancy or breastfeeding

12. Use of drugs or foods after study enrollment that are potent CYP3A4 inhibitors, including but not limited to atazanavir,
Table 1 Inclusion and exclusion criteria (Continued)

clarithromycin, indinavir, itraconazole, ketoconazole, nefazodone, nelfinavir, ritonavir, saquinavir, telithromycin, troleandomycin, voriconazole, and grapefruit or grapefruit juice

13. Use of drugs after study enrollment that are potent CYP3A4 inducers, including but not limited to carbamazepine, phenobarbital, phenytoin, rifabutin, rifampin, and St. John's wort

14. Use of drugs after study enrollment that are CYP3A4 substrates with narrow therapeutic indices, including but not limited to dihydroergotamine, ergotamine, pimozide, astemizole, cisapride, and terfenadine

15. Any other anticancer drugs including traditional Chinese medicine are prohibited

16. Evidence of active malignancy (other than NSCLC, non-melanoma skin cancer, localized cervical cancer, or localized and presumed cured prostate cancer) within the last 3 years

17. Other severe acute or chronic medical or psychiatric conditions, or laboratory abnormalities that would impart, in the judgment of the investigator, excess risk associated with study participation or study drug administration, and which would, therefore, make the patient inappropriate for entry into this study

18. Patients whom investigator judges to be inappropriate as participants

cgi-open-bin/ctr_e/ctr_view.cgi?recptno=R000035817). In this study, academic hospitals where at least one investigator-initiated clinical trial has been conducted in accordance with GCP standards will be recruited. Therefore, this study will be conducted at National Hospital Organization Kyushu Cancer Center, Okayama University Hospital, Hyogo Cancer Center, Nagoya University Hospital, Shizuoka Cancer Center, Kanazawa University Hospital, National Cancer Center Hospital, National Cancer Center Hospital East, and Hokkaido University Hospital, Japan.

\section{Eligibility criteria}

Table 1 shows key inclusion and exclusion criteria.

\section{Patient registration}

After eligibility criteria have been confirmed and informed consent has been obtained, eligible patients are registered, and the investigators initiate the planned treatment. On the consent form, participants will be asked if they agree to the use of their data should they choose to withdraw from the trial. Participants will also be asked for permission for the research team to share relevant data with people from the Universities taking part in the research or from regulatory authorities, where relevant. This trial involves collecting biological specimens for storage.

\section{Treatment plan}

Crizotinib $250 \mathrm{mg}$ BID will be administered orally at the same time on each day on a continuous daily dosing 
schedule unless sufficient toxicity develops to warrant dosing interruption/dose reduction. The cycles are defined as 28-day periods.

\section{Efficacy and safety evaluation}

Table 2 lists the time points for assessments of efficacy, adverse events, laboratory safety assessments (hematology, coagulation, and chemistry), physical examination, ECG, and tumor measurements. We will perform CTs and/or MRI scans every 8 weeks, and after 12 cycles, every 12 weeks for tumor assessments.

\section{Adverse events}

Adverse events will be classified based on the type, incidence, severity (graded by the National Cancer Institute Common Terminology Criteria for Adverse Events [CTCAE] Version 4.03), timing, seriousness, and relatedness. Adverse events are defined as the appearance of (or worsening of any pre-existing) undesirable sign, symptom, or medical condition that occurs after the first dose of the study treatment. Adverse event monitoring should be continued for at least 28 days following the last dose of the study

Table 2 Schedule of activities

\begin{tabular}{|l|c|c|c|c|c|c|c|}
\hline & \multirow{2}{*}{$\begin{array}{c}\text { Screening } \\
\text { Day }\end{array}$} & \multicolumn{2}{|c|}{ Cycle 1 } & $\begin{array}{c}\text { Cycle } \\
2+\end{array}$ & $\begin{array}{c}\text { End of } \\
\text { treatment }\end{array}$ & $\begin{array}{c}\text { Safety } \\
\text { follow-up }\end{array}$ & Follow-up \\
\cline { 3 - 8 } & $-14 \sim-1$ & $\begin{array}{c}\text { Day } \\
1\end{array}$ & $\begin{array}{c}\text { Day } \\
15\end{array}$ & $\begin{array}{c}\text { Day } \\
1\end{array}$ & & $\begin{array}{c}\text { 28 days } \\
\text { after last } \\
\text { dose }\end{array}$ & \\
\hline Treatment & & & & & & & \\
\hline Informed consent & $\bullet$ & & & & & & \\
\hline Patient characteristics & $\bullet$ & & & & & & \\
\hline ECOG-PS & $\bullet$ & $\bullet$ & $\bullet$ & $\bullet$ & $\bullet$ & & \\
\hline $\begin{array}{l}\text { Physical examinations/ } \\
\text { Vital signs }\end{array}$ & $\bullet$ & $\bullet$ & $\bullet$ & $\bullet$ & $\bullet$ & & \\
\hline Laboratory studies & $\bullet$ & $\bullet * * *$ & $\bullet$ & $\bullet$ & $\bullet$ & & \\
\hline ECG & $\bullet$ & & & & $\bullet$ & & $\bullet * * * *$ \\
\hline Chest X ray & $\bullet$ & & & $\bullet$ & $\bullet$ & & \\
\hline Pregnancy test & $\bullet *$ & & & $\bullet$ & $\bullet$ & & \\
\hline MET gene analysis & $\bullet$ & & & & & & \\
\hline Imaging assessment & $\bullet * *$ & & & $\bullet * * * *$ & $\bullet$ & & \\
\hline Adverse events & & & & & & & \\
\hline $\begin{array}{l}\text { Concomitant } \\
\text { medications/treatments }\end{array}$ & & & & & & & \\
\hline Survival follow-up & & & & & & & \\
\hline Allowance (Days) & $\bullet$ & & & & & & \\
\hline
\end{tabular}

* After Cycle 1 Day 1, pregnancy test should be performed when pregnancy is suspected

** Imaging assessments (Contrast CT of chest, abdomen, and pelvis and brain CT or MRI scan with contrast) will be performed at Day -28 to Day -1 prior to Cycle 1 Day 1 . Bone MRI is not required unless clinically indicated

*** Laboratory studies can be substituted with data carried out within 7 days

**** Every 8 weeks and, after 12 cycles, every 12 weeks, assessment will be performed using CT and/or MRI scan (+/- 7 days window). Chest X-rays are not required at the time of CT examination. Patients who discontinue prior to RECIST v1.1-defined progressive disease will continue with tumor assessments according to the protocol until disease progression is documented or initiation of additional anticancer therapy

***** Survival information will be collected every 3 months until death, loss to follow-up, or withdrawal of consent for survival. The investigator will collect survival information until 1 year after the last patient has enrolled into the study 
treatment or until the start of subsequent antineoplastic chemotherapy.

\section{Data collection and management}

All data will be recorded in a case report form (CRF) by investigators or clinical research coordinators. The completed CRFs will be submitted to the data center at the Five Rings Company. All study documents will be regarded as confidential. In the data center, the data will be stored and handled in a secure server maintaining the anonymity of participants.

\section{Statistical method}

In cohort 1 , an ORR of $20 \%$ is a clinically meaningful threshold for this study. We based the sample size on the exact binomial distribution. Assuming a null hypothesis of a $20 \%$ ORR and an alternative hypothesis of a $50 \%$ ORR, and a one-sided alpha error of 0.05 (one-side) and $80 \%$ power, the required evaluable patients with MET exon 14 skipping mutation-positive NSCLC is 19.

We expect cohort 2 to include mostly second- or later-line patients. The ORR of second-line single-agent chemotherapy in that clinical setting is approximately $10 \%$. The ORRs of second- or later-line treatment with PD-1/PD-L1 inhibitor for patients previously treated with platinum-based chemotherapy are 18-20\% [12-15].

We determined the sample size for cohort 2 based on the precision of ORR as a primary endpoint.

\section{Monitoring}

An independent Data and Safety Monitoring Committee has been established to assess the safety data when serious adverse events may occur. A qualified and independent auditor is appointed to audit the trial systems, and the trial was conducted before and during the study in accordance with a written procedure.

\section{Post-trial care}

This clinical trial will adopt clinical trial insurance; if serious harm occurs following the clinical trial, the affected participants will receive appropriate coverage.

\section{Discussion}

MET-TKIs being developed target the MET exon 14 skipping+ NSCLCs (NCT00585195, NCT02864992, NCT02414139). With the safety data of crizotinib firmly established, we expect its imminent approval as the first MET-TKI.

A sensitive, reliable molecular test should be incorporated into the trial to obtain a compendium of approved diagnostics for MET alterations. Therefore, we allowed enrollment of patients positive for MET exon 14 skipping mutation or high MET copy number of seven or greater, as determined by a validated RT-PCR and/or
NGS assay by a designated central testing laboratory (LC-SCRUM) in this trial.

If crizotinib demonstrates a meaningful clinical benefit in Japanese patients with MET exon 14 skipping mutation-positive NSCLC in this trial, our results will help crizotinib receive approval for these patients in Japan.

In conclusion, our study will evaluate the efficacy and safety of oral single-agent crizotinib for the treatment of advanced MET exon 14 skipping mutation-positive or MET high gene copy number-positive NSCLC.

\section{Trial status}

The trial has been registered at the University Hospital Medical Information Network Clinical Trials Registry (registration number: UMIN000031623; registration date: 3 March 2018; https://www.umin.ac.jp/ctr/index.htm). Recruitment started in March 2018 and is expected to finish in May 2020. The protocol version number is 1.0.

\section{Abbreviations}

ALK: Anaplastic lymphoma receptor tyrosine kinase; BID: b.i.d. oral dose; DCR: Disease control rate; DR: Duration of response; HGF: Hepatocyte growth factor; MET: Mesenchymal-epithelial transition; NGS: Next-generation sequencing; NSCLC: Non-small cell lung cancer; ORR: Objective response rate; OS: Overall survival; PFS: Progression-free survival; RECIST: Response Evaluation Criteria in Solid Tumors; TKI: Tyrosine kinase inhibitor; ULN: Upper limit of normal; RT-PCR: Reverse Transcription Polymerase Chain Reaction; OCA: Oncomine Comprehensive Assay

\section{Acknowledgements}

The authors would like to thank the patients, their families, and all the investigators who will participate in the present study.

\section{Authors' contributions}

$\mathrm{KN}$ drafted the manuscript. KN, MS, and KY contributed equally to this work. $M S, K N, T S, K O, M M, H H, J S, H M, S Y, M S, S M, K G$, and KY designed the study. MS is responsible for planning the data analysis and analyzing the data resulting from the trial. All named authors adhere to the authorship guidelines of Trials. All authors have agreed to publication. All authors read and approved the final manuscript.

\section{Funding}

This study is conducted by the Five Rings company and financially supported by the Practical Research for Innovation Cancer Control from Japan Agency for Medical Research and Development, AMED. The Five Rings company will conduct study coordination, data management, monitoring, statistical analysis, and writing of the report according to relevant operating procedures. AMED will play no part in the study design; collection, management, analysis, and interpretation of data; writing of the report; and the decision to submit the report for publication.

\section{Availability of data and materials}

We will disseminate the results of this clinical trial widely through conference presentations and publications in relevant journals. All evaluation forms, reports, and other records will be identified in a manner designed to maintain participant confidentiality. All records will be kept in a secure storage area with limited access. The data from this clinical trial will not be shared. The Investigator and study site staff involved with this study may not disclose or use for any purpose other than performance of the study, any data, record, or other unpublished, confidential information disclosed to those individuals for the purpose of the study. The Co-MET study does not involve collecting biological specimens for storage.

\section{Ethics approval and consent to participate}

The study was approved by the IRB at the National Hospital Organization Kyushu Cancer Center on 7 March 2018 (*21, protocol version 1.0), Okayama 
University Hospital on 15 May 2018 (M300206), Hyogo Cancer Center on 9 May 2018 (HCC227), Nagoya University Hospital on 10 September 2018 (302007), Shizuoka Cancer Center on 24 May 2018 (no reference), Kanazawa University Hospital on 13 June 2018 (9016), National Cancer Center Hospital on 23 May 2018 (T4552), National Cancer Center Hospital East on 23 May 2018 (K0715), and Hokkaido University Hospital on 22 May 2018 (H30-5). Any protocol modification will be approved by the IRBs before its implementation. This study will be conducted in compliance with the study protocol, the Helsinki declaration and the Ministerial Ordinance on Good Clinical Practice (GCP) for Drugs. All participants will give written informed consent. Central ethical approval has been confirmed from the IRB at the National Hospital Organization Kyushu Cancer Center (ref approval no. *21), and we will not begin recruiting at other centers in the trial until local ethical approval has been obtained. Written informed consent will be provided by patients before enrollment. The informed consent documentation is are available from the corresponding author on request.

\section{Consent for publication}

Not applicable.

\section{Competing interests}

KN and SM have received Honoraria from Pfizer Japan. TS has received Honoraria and research grants from Pfizer Japan. HM has received personal fees from Pfizer Japan. KG has received Honoraria and research funding from Pfizer Japan. KY has received research funding from Pfizer Japan. All other authors declare that they have no conflicts of interest.

\section{Author details}

${ }^{1}$ Cancer Biostatistics Laboratory, Clinical Research Institute, National Hospital Organization Kyushu Cancer Center, Fukuoka, Japan. ${ }^{2}$ Department of Biostatistics, Yamaguchi University Graduate School of Medicine, 1-1-1 Minamikogushi, Ube, Yamaguchi 755-8505, Japan. ${ }^{3}$ Department of Thoracic Oncology, National Hospital Organization Kyushu Cancer Center, Fukuoka, Japan. ${ }^{4}$ Department of Thoracic Oncology, National Cancer Center Hospital East, Chiba, Japan. ${ }^{5}$ Department of Allergy and Respiratory Medicine, Okayama University Hospital, Okayama, Japan. ${ }^{6}$ Department of Respiratory Medicine, Nagoya University Graduate School of Medicine, Nagoya, Japan. 7Department of Thoracic Oncology, National Cancer Center Hospital, Tokyo, Japan. ${ }^{8}$ First Department of Medicine, Hokkaido University Hospital, Hokkaido, Japan. ${ }^{9}$ Division of Thoracic Oncology, Shizuoka Cancer Center, Shizuoka, Japan. ${ }^{10}$ Division of Medical Oncology, Cancer Research Institute, Kanazawa University, Kanazawa, Japan. ${ }^{11}$ Department of Thoracic Oncology, Hyogo Cancer Center, Hyogo, Japan.

Received: 4 December 2019 Accepted: 2 March 2020

Published online: 30 March 2020

\section{References}

1. Maemondo M, Inoue A, Kobayashi K, Sugawara S, Oizumi S, Isobe H, et al. Gefitinib or chemotherapy for non-small-cell lung cancer with mutated EGFR. N Engl J Med. 2010;362:2380-8.

2. Mitsudomi T, Morita S, Yatabe Y, Negoro S, Okamoto I, Tsurutani J, et al. Gefitinib versus cisplatin plus docetaxel in patients with non-small-cell lung cancer harbouring mutations of the epidermal growth factor receptor (WJTOG3405): an open label, randomised phase 3 trial. Lancet Oncol. 2010; 11:121-8.

3. Solomon BJ, Mok T, Kim DW, Wu YL, Nakagawa K, Mekhail T, et al. First-line crizotinib versus chemotherapy in ALK-positive lung cancer. N Engl J Med. 2014;371:2167-77.

4. Kohno T, Nakaoku T, Tsuta K, Tsuchihara K, Matsumoto S, Yoh K, et al. Beyond ALK-RET, ROS1 and other oncogene fusions in lung cancer. Transl Lung Cancer Res. 2015:4:156-64.

5. Kawakami H, Okamoto I, Okamoto W, Tanizaki J, Nakagawa K, Nishio K. Targeting MET amplification as a new oncogenic driver. Cancers (Basel). 2014;6:1540-52.

6. Kong-Beltran M, Seshagiri S, Zha J, Zhu W, Bhawe K, Mendoza N, et al. Somatic mutations lead to an oncogenic deletion of met in lung cancer. Cancer Res. 2006;66:283-9.

7. Drilon A, Camidge DR, Ou SH, Clark JW, Socinski MA, Weiss J, et al. Efficacy and safety of crizotinib in patients (pts) with advanced MET exon 14-altered non-small cell lung cancer (NSCLC). J Clin Oncol. 2016;34:108.
8. Frampton GM, Ali SM, Rosenzweig M, Chmielecki J, Lu X, Bauer TM, et al. Activation of MET via diverse exon 14 splicing alterations occurs in multiple tumor types and confers clinical sensitivity to MET inhibitors. Cancer Discov. 2015:5:850-9.

9. Paik PK, Drilon A, Fan PD, Yu H, Rekhtman N, Ginsberg MS, et al. Response to MET inhibitors in patients with stage IV lung adenocarcinomas harboring MET mutations causing exon 14 skipping. Cancer Discov. 2015;5:842-9.

10. Camidge DR, Ou S-HI, Shapiro G, Otterson GA, Villaruz LC, Villalona-Calero MA, et al. Efficacy and safety of crizotinib in patients with advanced c-METamplified non-small cell lung cancer (NSCLC). J Clin Oncol. 2014;32:8001.

11. Ou SH, Kwak EL, Siwak-Tapp C, Dy J, Bergethon K, Clark JW, et al. Activity of crizotinib (PF02341066), a dual mesenchymal-epithelial transition (MET) and anaplastic lymphoma kinase (ALK) inhibitor, in a non-small cell lung cancer patient with de novo MET amplification. J Thorac Oncol. 2011;6:942-6.

12. Borghaei H, Paz-Ares L, Horn L, Spigel DR, Steins M, Ready NE, et al. Nivolumab versus docetaxel in advanced nonsquamous non-small-cell lung cancer. N Engl J Med. 2015;373:1627-39.

13. Brahmer J, Reckamp KL, Baas P, Crinò L, Eberhardt WE, Poddubskaya E, et al. Nivolumab versus docetaxel in advanced squamous-cell non-small-cell lung cancer. N Engl J Med. 2015;373:123-35.

14. Herbst RS, Baas P, Kim D-W, Felip E, Pérez-Gracia JL, Han JY, et al. Pembrolizumab versus docetaxel for previously treated, PD-L1-positive, advanced non-small-cell lung cancer (KEYNOTE-010): a randomised controlled trial. Lancet. 2016:387:1540-50.

15. Rittmeyer A, Barlesi F, Waterkamp D, Park K, Ciardiello F, Von Pawel J, et al. Atezolizumab versus docetaxel in patients with previously treated nonsmall-cell lung cancer (OAK): a phase 3, open-label, multicentre randomised controlled trial. Lancet. 2017;389:255-65.

\section{Publisher's Note}

Springer Nature remains neutral with regard to jurisdictional claims in published maps and institutional affiliations.

\section{Ready to submit your research? Choose BMC and benefit from:}

- fast, convenient online submission

- thorough peer review by experienced researchers in your field

- rapid publication on acceptance

- support for research data, including large and complex data types

- gold Open Access which fosters wider collaboration and increased citations

- maximum visibility for your research: over $100 \mathrm{M}$ website views per year

At BMC, research is always in progress.

Learn more biomedcentral.com/submissions 